in Africa, where resistance to chloroquine is decreasing in many countries and where the combination of piperaquine and dihydroartemisinin remains highly effective ${ }^{12}$. We can look forward to further studies of PfCRT, including visualization of the drug-bound and open-to-cytoplasm structural conformations, which will further explain the effects of resistance mutations and might help to identify drugs that circumvent resistance. For now, we can appreciate the insights gained from Kim and colleagues' beautiful marriage of structure, biochemistry, genetics and parasitology, and particularly from the first atomic-resolution structure of PfCRT - the fine-tuned, resistance-mediating machine of malaria parasites.

Leann Tilley is in the Department of Biochemistry and Molecular Biology, University of Melbourne, Parkville, Victoria 3010, Australia. Philip J. Rosenthal is in the Department of Medicine, University of California, San Francisco, San Francisco, California 94143 USA.

e-mails: Itilley@unimelb.eud.au;

philip.rosenthal@ucsf.edu

1. World Health Organization. World Malaria Report 2018 (2018).

2. Haldar, K., Bhattacharjee, S. \& Safeukui, I. Nature Rev Microbiol. 16, 156-170 (2018)

3. Kim, L. et al. Nature 576, 315-320 (2019).

4. Goldberg, D. E. Curr. Top. Microbiol. Immunol. 295 , 275-291 (2005).

5. van der Pluijm, R. W. et al. Lancet Infect. Dis. 19, 952-961 (2019).

6. Ross, L. S. et al. Nature Commun. 9, 3314 (2018).

7. Fidock, D. A. et al. Mol. Cell 6, 861-871 (2000).

8. Summers, R. L. et al. Proc. Natl Acad. Sci. USA 111, E1759-1767 (2014).

9. Martin, R. E. \& Kirk, K. Mol. Biol. Evol. 21, 1938-1949 (2004). 10. Nogales, E. \& Scheres, S. H. Mol. Cell 58, 677-689 (2015).

11. Pelleau, S. et al. Proc. Natl Acad. Sci. USA 112, 11672-11677 (2015).

12. Conrad, M. D. \& Rosenthal, P. J. Lancet Infect. Dis. 19, e338-e351 (2019).

This article was published online on 27 November 2019.

\title{
A step closer to the Sun's secrets
}

\section{Daniel Verscharen}

NASA's Parker Solar Probe is currently making a series of

close encounters with the Sun. Initial observations from the spacecraft have improved our understanding of both the Sun and its environment. See p.223, p.228, p.232 \& p.237

Although the Sun is quite near to us compared with other stars, it has always kept intriguing and fundamental scientific secrets from us. For instance, we still don't know how the solar corona - the Sun's outermost atmosphere - maintains temperatures in excess of one million kelvin, whereas the visible surface has temperatures of just below $6,000 \mathrm{~K}$ (ref. 1). The corona produces the solar wind, an outflow of plasma particles (free ions and electrons) that expands into the space between the planets. In 2018, NASA launched the Parker Solar Probe ${ }^{2}$ (PSP) with the aim of identifying the mechanisms behind the heating of the corona and the acceleration of the solar wind. Four papers in Nature ${ }^{3-6}$ report the first results from the PSP.

The measurements from the PSP were taken when the spacecraft was as close as 24 million kilometres to the Sun (for comparison, the average distance between Mercury and the Sun is about 58 million kilometres). They show that the solar wind near the Sun is much more structured and dynamic than it is at Earth (Fig. 1). On page 237, Bale et al. ${ }^{3}$ present measurements of the direction and close encounter with the Sun. strength of the Sun's magnetic field, which is dragged out into space by the solar wind. The authors find rapid reversals in the direction of the field that last for only minutes.
Although some similar magnetic structures have been seen before ${ }^{7}$, the large amplitude and the high occurrence rate of these reversals are surprising. In fact, the nature of these structures remains unknown.

Bale and colleagues also report that the PSP's sensors detected fluctuations in the local electric and magnetic fields in the solar wind that are larger than those detected near Earth. These fluctuations can be generated by turbulence in the solar wind or by plasma instabilities that are driven by ions or electrons. The presence of such fluctuations suggests that plasma instabilities have a much larger effect on the dynamics and energetics of the solar wind than previously expected.

On page 228 , Kasper et al. ${ }^{4}$ present observations of the Sun's plasma ions and electrons. They find that the reversals in the Sun's magnetic field are often associated with localized enhancements in the radial component of the plasma velocity (the velocity in the direction away from the Sun's centre). The authors use the extremely clear signal of the solar wind's strahl - a collimated and fast beam of electrons that stream along the magnetic field - to study the field's geometry and configuration. This method leads Kasper and colleagues to interpret the magnetic-field reversals as travelling $\mathrm{S}$-shaped bends in the field lines coming from the Sun.

These authors also report a surprisingly large azimuthal component of the plasma velocity (the velocity perpendicular to the radial direction). This component results from the force with which the Sun's rotation slingshots plasma out of the corona when the plasma is released from the coronal magnetic field - much like a spinning hammer-thrower slingshots the hammer when releasing it from their hands. However, the reason for the large observed value of the azimuthal velocity is currently unclear.

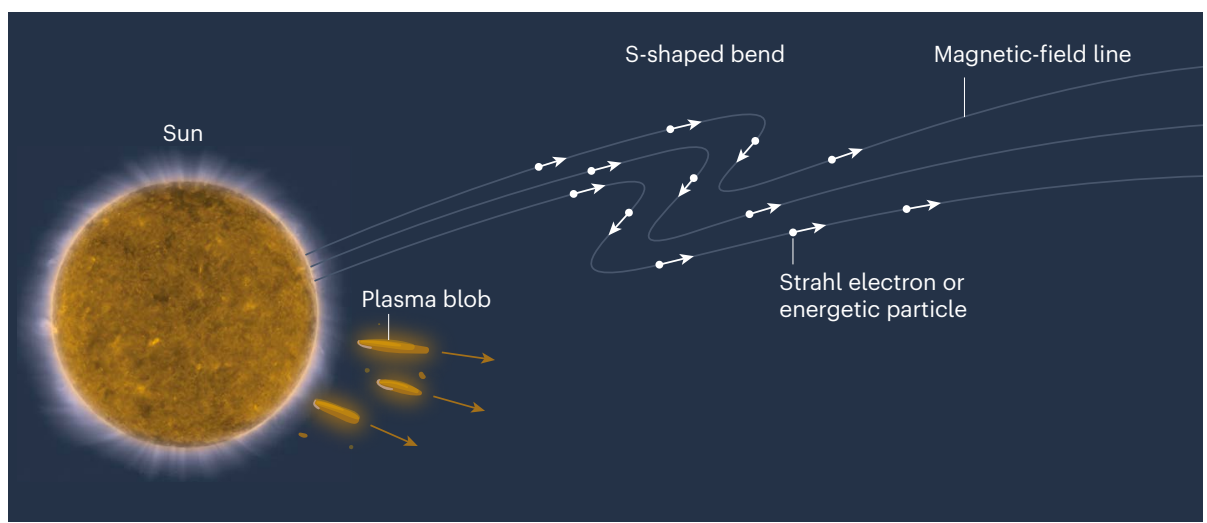

Figure 1 | The near-Sun environment. The Sun's outermost atmosphere generates an outflow of plasma particles (ions and electrons) called the solar wind. 'Strahl' electrons and energetic particles in the wind stream along the Sun's magnetic-field lines. Four papers ${ }^{3-6}$ report observations from the Parker Solar Probe (PSP), which is currently in orbit around the Sun. The PSP data suggest that the field lines contain S-shaped bends and that the Sun releases blobs of plasma that form part of the young solar wind. The ultraviolet-light image of the Sun was taken by NASA's Solar Dynamics Observatory on the day that the PSP made its first 
On page 223, McComas et al. ${ }^{5}$ study detections of energetic ions and electrons, some of which are observed more often in the region just outside the corona than they are near Earth. These particles are accelerated by flares (eruptions of radiation) in the corona or by shock waves associated with coronal mass ejections (eruptions of plasma), which travel through interplanetary space. The authors identify particles corresponding to both types of source region.

Because energetic particles travel along the Sun's magnetic field, the difference in the time at which fast and slow particles arrive at the PSP can be used to estimate the path length of their trajectory along the field. McComas and colleagues find that this path length is longer than expected, which suggests that the magnetic field has a more complicated geometry than assumed. This finding could be accounted for by the S-shaped magnetic-field reversals.

The imaging instrument on board the PSP makes remote observations of light scattered by electrons and dust near the Sun. On page 232, Howard et al. ${ }^{6}$ report that the intensity of the dust-scattered light decreases with distance from the Sun in almost the same way as it does when observed from Earth. However, the authors find some preliminary evidence for the existence of a hypothesized dust-free zone ${ }^{8}$ near the Sun that has not been detected before. The detailed images from the PSP also show spatial variations in the solar wind that are consistent with variations in the Sun's magnetic field on its surface, and reveal small blobs of plasma that are ejected from the Sun and form part of the young solar wind.

These four papers show that, by going into an unexplored region of the Solar System, the PSP has already made great discoveries. In the near future, it will be important to combine all the available sources of information to develop a deeper understanding of the physics of the Sun and the solar wind. For instance, researchers should combine the measurements of the electric and magnetic fields with detailed observations of the plasma particles to determine how fields and plasma interact and drive instabilities ${ }^{9}$. They must also study the large azimuthal flow velocity further to confirm whether it is a persistent feature or just a one-time exception during these initial PSP measurements.

The use of magnetic-field models will enable scientists to learn more about the path of energetic particles between the Sun and the PSP, and, in turn, about space weather - the effects of the Sun and the solar wind on Earth and human technology. These energetic-particle studies must also be linked with remote observations of the Sun's surface and the corona. Examining the potential presence of the dust-free zone near the Sun must be another short-term goal, but might have to wait for closer approaches of the PSP to the Sun in the future.

It is expected that PSP data will guide our understanding of the Sun and the solar wind for many years. New models and theories will be motivated by the spacecraft's discoveries, and this knowledge will be transferable to other stars and astrophysical plasmas throughout the Universe. After all, the Sun is the only star that we can study up close using spacecraft. The orbit of the PSP will bring the spacecraft even closer to the Sun in the coming years, to just over 6 million kilometres from the surface ${ }^{2}$. During this time, the Sun will transition into a more active phase of its 11-year cycle, so we can expect even more-exciting results soon.

In 2020, the European Space Agency will launch the Solar Orbiter mission ${ }^{10}$. Although this spacecraft will not go quite as close to the Sun as will the PSP, its more extensive suite of scientific instruments will be used in combination with the PSP to reveal key information about the Sun. For example, Solar Orbiter will measure the elemental composition and charge states of ions and will take photographs of the Sun in different wavelengths of light. These joint measurements will certainly close some of the remaining gaps in our knowledge of the Sun and the solar wind. For now, however, the Sun has proved again that it still holds more secrets for us to discover.

Daniel Verscharen is at the Mullard Space Science Laboratory, University College London, Dorking RH5 6NT, UK.

e-mail: d.verscharen@ucl.ac.uk

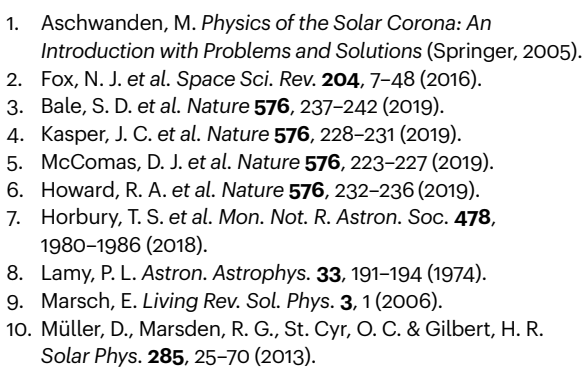

This article was published online on 4 December 2019.

\section{Electronics}

\section{Graphene sees the light in wearablesensors}

\section{Deji Akinwande \& Dmitry Kireev}
Graphene coated with nanoparticles has been used to make
wearable light sensors that measure the human pulse and blood oxygen levels from ambient light passing through tissue, offering a potential platform for health-care monitoring.

The popularity of wearable technology has risen enormously, with the US market projected to be in the tens of billions of dollars by 2022 (see go.nature.com/33tcein). However, the effectiveness of the most common wearable devices is hindered by the physical specifications of their components: although the device is often embedded in a flexible soft shell, the main parts, such as the sensors and electronics, are still rigid ${ }^{1,2}$. Now, writing in Science Advances, Polat et al. ${ }^{3}$ report a class of truly flexible, transparent wearable device that is based on graphene covered with a layer of semiconducting nanoparticles known as quantum dots. Impressively, the devices measure various vital signs using only ambient light as a signal.

Materials that are just one or a few atoms thick are said to be two-dimensional. The best-known example is graphene, which consists of single sheets of carbon atoms arranged in a hexagonal lattice. $2 \mathrm{D}$ materials in general, and graphene in particular, have tremendous potential for the development of next-generation wearable, soft biosensors because they combine electrical conductivity, optical transparency and mechanical flexibility with outstanding biocompatibility ${ }^{4}$ and stability to biological electrolytes. Graphene-based tattoo-like devices ${ }^{5}$ have previously been used to record human health signals such as heart rhythm, skin hydration and body temperature. Their outstanding performance is associated with the subnanometre thickness of graphene, which allows it to bend and stretch with the skin, without affecting the sensor performance.

Polat et al. have now expanded the functionality of graphene in wearable devices by depositing light-sensitive quantum dots made of the semiconductor lead(II) sulfide (PbS) onto the graphene layer. When illuminated, the quantum dots generate pairs of charged particles: negatively charged electrons and 